\title{
Red supergiant stars in blue compact dwarf galaxies: The case of Tol $0610-387^{\star}$
}

\author{
V. Doublier ${ }^{1,2}$, J. M. Mas-Hesse ${ }^{3}$, A. Caulet $^{5}$, and D. Kunth ${ }^{4}$ \\ 1 European Southern Observatory, Karl Schwarzchild str. 2, 85748 Garching, Germany \\ 2 Observatoire de Marseille and Institut Cassendi, 2 place Le Verrier, 13004 Marseille, France \\ 3 LAEFF-INTA, Apdo. 50727, 28080 Madrid, Spain \\ 4 Institut d'Astrophysique de Paris, 98 bis Bd. Arago, 75014 Paris, France \\ 5 Dept. of Astronomy, University of Illinois at Urbana-Champaign, 1002 West Green Street Urbana, \\ Ill 61801, USA
}

Received 30 March 1999 / Accepted 1 March 2001

\begin{abstract}
We have investigated the stellar content of the blue compact dwarf galaxies (BCDG) Tol 0610-387 by combining near infrared and optical images, complemented by spectroscopic data. The color maps, combined with the $\mathrm{H} \alpha$ image allowed us to identify clusters possibly dominated by red supergiant stars (RSG) associated with the current ongoing starburst episode. The analysis shows that the local colors and H $\alpha$ emission, when compared with the predictions from population synthesis models, are consistent with an aging stellar population of around 10-15 Myrs dominated by red supergiant stars. This galaxy could therefore become a preferred target for the spectroscopic confirmation and analysis of its RSG population. Since the presence of such stars in BCDGs is seldom observed, we discuss the implications of RSG detection for the study of the starburst dynamics and evolution.
\end{abstract}

Key words. galaxies: photometry - galaxies: stellar content - galaxies: compacts - galaxies: dwarfs - galaxies: evolution - galaxies: fundamental parameters

\section{Introduction}

Blue Compact Dwarf Galaxies are dominated by very intense and short-lived episodes of massive star formation. During these episodes, $10^{4}$ to $10^{6}$ solar masses of gas are turned into stars with initial masses up to about $100 M_{\odot}$ (Meurer et al. 1995; Massey et al. 1995; Mas-Hesse 1999). While the massive stars seem to form almost co-evally (within few Myrs in any case), it is unclear whether there is a low mass cut-off in the IMF or not. The strength of the starburst (measured by the absolute $\mathrm{H} \beta$ luminosity) seems to be linked to the total mass of the object, since this absolute $\mathrm{H} \beta$ emission and its velocity dispersion are correlated through a power law (Melnick et al. 1987); this relation is apparently valid for all starbursting galaxies (with velocity dispersions smaller than $60 \mathrm{~km} \mathrm{~s}^{-1}$ ). Nevertheless, the ionized gas surrounding these star-forming regions can become highly turbulent because of stellar winds, interaction

\footnotetext{
Send offprint requests to: V. Doublier,

e-mail: vdoublie@eso.org

* Based on observations made at $2.2 \mathrm{~m}$ Telescope and $1.54 \mathrm{~m}$ Danish telescope at La Silla, (ESO).
}

with shells and frequent supernova explosions (AmbrocioCruz et al. 1997; Izotov et al. 1996; Chu \& Kennicutt 1994; Roy \& Belley 1993). Therefore the velocity dispersion may not trace primarily the general motion of stars in individual HII regions, and can even be disconnected from the global dynamics of the host galaxy. An example of this disconnection was found by Lequeux et al. (1995) and Legrand et al. (1997) in Haro 2, where the gas is outflowing from the star formation region at about $200 \mathrm{~km} \mathrm{~s}^{-1}$.

Therefore, the true velocity dispersion of the young stars can be traced only by analyzing stellar lines, like the Calcium triplet absorption feature in red supergiant stars (RSG). RSGs are not expected to appear in starburst regions until 6 Myrs after the onset of the burst (Cerviño \& Mas-Hesse 1994), i.e., when the ionizing power of the cluster, and therefore the emission lines, begin to fade. Since the selection of BCDGs is mainly based on their emission lines, there is a severe bias against the detection of objects with star formation regions old enough to be dominated by a population of RSG stars. This explains the low detection rate of these stars in BCDGs. While RSGs have been detected by $B V R$ photometry and follow-up 
spectroscopy based on the Ca Triplet in the near-infrared in Local Group Galaxies have been carried out (Massey 1998), the presence of RSGs has been established only in a few star-forming dwarf galaxies. Red supergiants have been detected in He2-10 (Zinnecker 1987), and in Tol 3 and Tol 1457-262 (Campbell \& Terlevich 1984) using CO absorption lines in the near infrared. More direct detections in the extreme low abundance blue compact dwarf galaxy I Zw 18 have been reported by Hunter \& Thronson (1995).

RSGs can be detected and located by analyzing the continuum colors of regions where star formation is known to have taken place in the last $20 \mathrm{Myr}$. Once detected, spectroscopic follow-up makes it possible to measure properly the velocity dispersion using the Calcium triplet. We followed this approach on a sample of Blue Compact Galaxies, with potential positive detection of RSGs.

These BCDGs will be used to study the kinematics of the stars in the starburst region, by measuring the Calcium triplet associated to the RSG population, assuming that these young stars have kept the motions of the original clouds within the galaxy potential (the diffusion time scale for these stars is larger than their evolution time scale by at least an order of magnitude). Moreover, the starbursts in the BCDGs represent a large fraction of their stellar content, hence this would help understanding the dynamics of these clouds with respect to the host galaxy. In Mrk 996 for instance, the star formation occurs in a small disk (Thuan et al. 1996; Doublier et al. 2001), regardless of the apparent structure of the host galaxy that shows strong evidence for being a pure elliptical (Doublier et al. 1997). The relations between the dynamics of the starburst and the host galaxy could give strong indication on the origin of the star formation triggering factor, either by slow gas accretion during gravitational interaction (larger or smaller galaxy fly-by, or in-falling HI clouds), or more dramatically during major mergers.

In this work we analyze in detail the properties of the most certain candidate to contain RSGs, Tol 0610-387.

We present in Sect. 2 our observational data, and discuss in Sect. 3 the constraints we can derive on the stellar population by comparing with the predictions of evolutionary synthesis models. Finally, in Sect. 4 we summarize our conclusions.

\section{Observations}

\subsection{Data}

In December 1995, we obtained $J, H$ and $K \mathrm{~s}$ band images of the Blue Compact Dwarf Galaxy Tol 0610-387 during a campaign of IR imaging observations at the $2.2 \mathrm{~m}$ telescope in La Silla (Chile). The instrument was IRAC2, a $256 \times 256$ NICMOS3 camera; we chose a pixel size of $0^{\prime \prime} .507$ to have a field of view of $2^{\prime} \times 2^{\prime}$. The BCDGs observed are about $60^{\prime \prime}$ (or less) in diameter at $B=25 \mathrm{mag} \operatorname{arcsec}^{-2}$ which corresponds to magnitude fainter than $22 \mathrm{mag} \operatorname{arcsec}^{-2}$ in $K$ at best. The detection

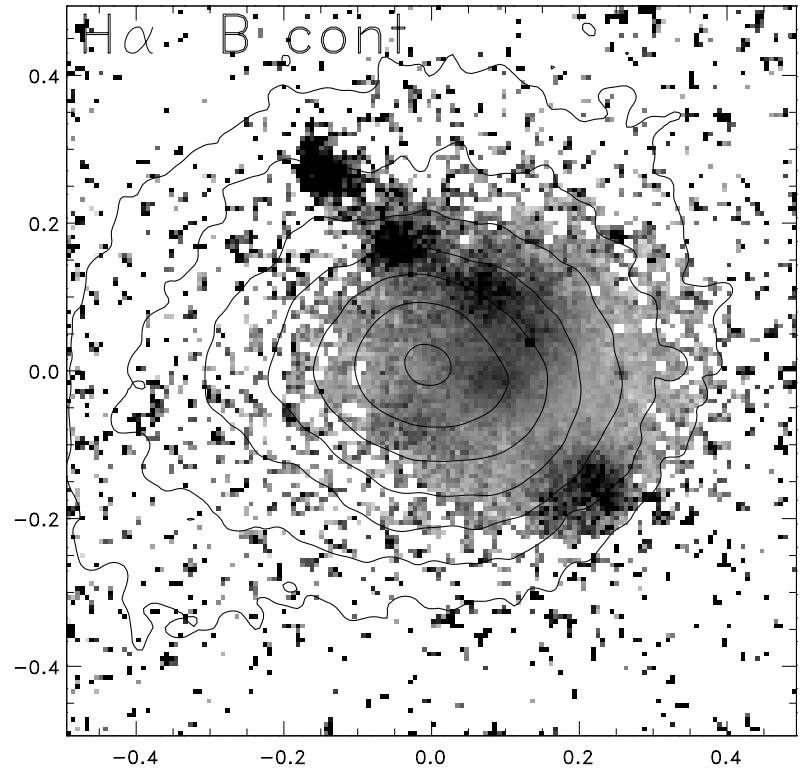

Fig. 1. Tol 0610-387 H $\alpha$ map. Note the different knots corresponding to strong $\mathrm{H} \alpha$ emission, along the SW-NE direction. The contours represent the continuum in the $B$ band in units of $S / N(1000,800,400,200,150,100,50)$ the lowest value corresponds to $24 \mathrm{mag} \operatorname{arcsec}^{-2}$. Note that the maximum of continuum emission does not correspond to any of the local maxima of $\mathrm{H} \alpha$ emission. The $\mathrm{H} \alpha$ emission is off-center with respect to the center of the galaxy. The spatial scale of all images is given in fraction of arcminutes. North is up and East is left. The (0:0) location corresponds to the same center defined by the maximum of emission in all broad bands $B, I$ and $R$. The regions on the $\mathrm{H} \alpha$ map corresponding to $S / N$ lower than 50 in the $B$ band are processing artifacts and should not be taken into account.

limit of IRAC2 is $22 \mathrm{mag} \operatorname{arcsec}^{-2}$ in $K \mathrm{~s}$ for one hour of total exposure time which in the case of Tol 0610-387 corresponds to a diameter of $20^{\prime \prime}$. Thus, the size of the field allowed us to use the in-field chopping techniques in order to optimize overheads and increase the effective exposure times. The total integration time was: $27 \mathrm{~min}$ in $J, 28 \mathrm{~min}$ in $H$ and $80 \mathrm{~min}$ in $K$ (this gives a signal to noise ratio of 3 at $\left.K=22 \mathrm{mag} \operatorname{arcsec}^{-2}\right)$. During the same period, we also obtained $B$ and $R$ band images at the $1.54 \mathrm{~m}$ Danish Telescope in La Silla, Chile. The instrument was DFOSC equipped with a $2 k \times 2 k$ Loral UV-floaded CCD. The pixel size was 0 ". 38 . The total integration time was $60 \mathrm{~min}$ in $B$ and $30 \mathrm{~min}$ in $R$. The quality of the data allowed us to go down to $1 \%$ of the sky background, i.e. $S / N=3$ at $B=25 \mathrm{mag} \operatorname{arcsec}^{-2}$. The integrated magnitudes were extrapolated from the curve of growth derived from the surface photometry. In addition, we obtained both $\mathrm{H} \alpha$ and $I$ images of Tol 0610-387 using the NTT equipped with SUSI ( 0 '. $13 /$ pixel) with a field of view of $1^{\prime} \times 1^{\prime}$.

All the broad band $B, R, I, J, H$, and $K$ s images were flux calibrated, using standard stars from the Landolt equatorial standards Landolt (1992), and the standard stars from the Carter system respectively (for detailed 


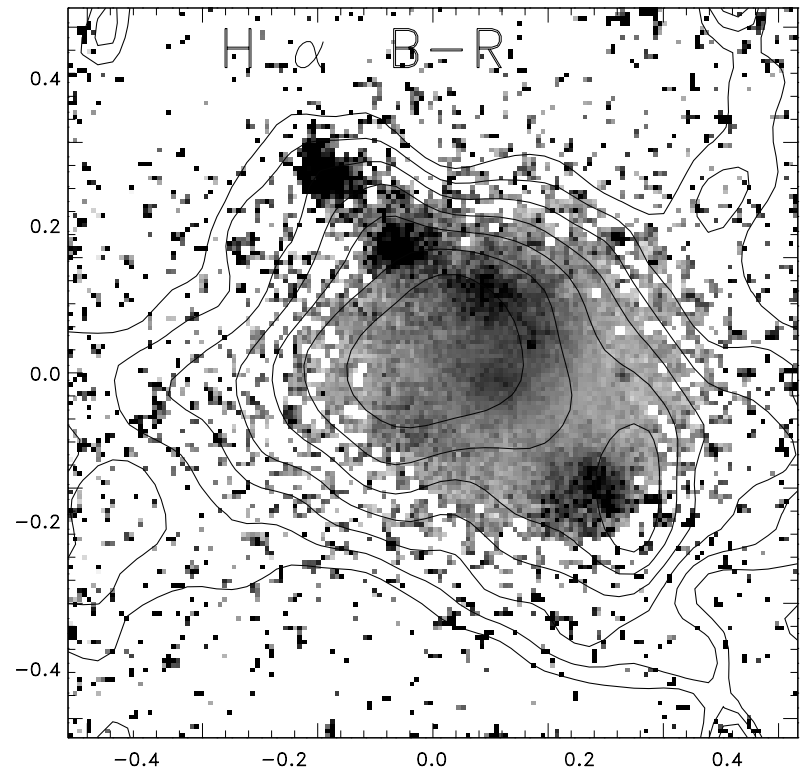

Fig. 2. Tol 0610-387 H $\alpha$ map with the contours of the $B-R$ color map superimposed. The outermost contour corresponds to a $B-R=3$. The contours are spaced by decreasing $0.05 \mathrm{mag}$. Note that the minimum of the $B-R$ color does not correspond to the maximum of the $\mathrm{H} \alpha$ emission; the $\mathrm{H} \alpha$ emission appears to surround the regions of minimum $B-R$.

Table 1. Photometric integrated magnitudes, and spectroscopic and derived data on Tol 0610-387. Spectroscopic data from Terlevich et al. (1991) were obtained through a circular aperture $8^{\prime \prime}$ in diameter and are accurate to around $10 \%$. The broad band magnitudes are corrected for the Galactic extinction taken from Burstein \& Heiles (1982).

\begin{tabular}{ll|ll|ll}
\hline \multicolumn{2}{c|}{ Optical (mag) } & \multicolumn{2}{|c}{ IR (mag) } & \multicolumn{2}{c}{ O lines* } \\
\hline$B$ & $15.78 \pm 0.02$ & $J$ & $12.87 \pm 0.05$ & {$[\mathrm{OII}] 3727 / \mathrm{H} \beta$} & 4.512 \\
$R$ & $14.16 \pm 0.01$ & $H$ & $12.03 \pm 0.08$ & {$[\mathrm{OIII}] 4959 / \mathrm{H} \beta$} & 0.976 \\
$I$ & $14.90 \pm 0.03$ & $K$ & $12.32 \pm 0.07$ & {$[\mathrm{OIII}] 5007 / \mathrm{H} \beta$} & 3.118 \\
\hline$W(\mathrm{H} \beta)^{*}$ & & \multicolumn{1}{c}{$\begin{array}{l} \\
\log [F(\mathrm{H} \beta)]^{*}\end{array}$} & $-14.60 \mathrm{erg} \mathrm{s} \mathrm{cm}^{-2}$ \\
$12+\log (\mathrm{O} / \mathrm{H})^{*}$ & & $7.8 / 8.4$ & \\
$E(B-V)_{\text {Gal }}$ & & 0.062 & \\
\hline
\end{tabular}

(*) From spectroscopic data.

description (see Doublier et al. 1997, 1999). The H $\alpha$ images are not flux calibrated. At the time the images were obtained, we could not take any narrow-band continuum to subtract from the $\mathrm{H} \alpha$ image, nor standard stars. So, the $\mathrm{H} \alpha$ "map" was constructed by subtracting the almost featureless continuum $I$ image. Since both images were taken with the same camera and telescope, we simply skysubtracted the frames and realigned them, before combining them. In the present article, we are not interested in the $\mathrm{H} \alpha$ flux, but more in the morphology and spatial distribution of the $\mathrm{H} \alpha$ emission.

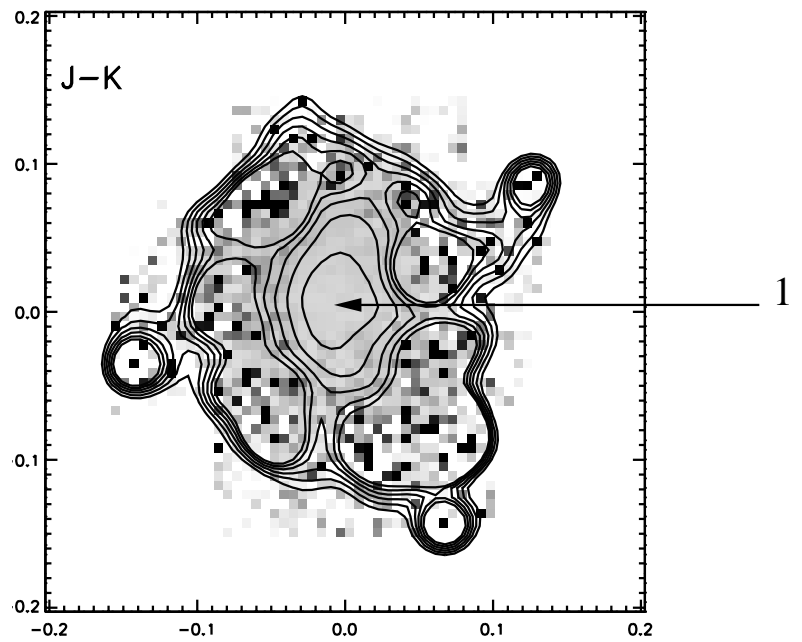

Fig. 3. Tol 0610-387 $J-K$ image. The outermost contour corresponds to $J-K=0.63$ The $J-K$ contour levels are spaced by 0.1 mag. Region 1 is identified: see Sect. 2.2 .

The surface photometry has been complemented with spectroscopic data from Terlevich et al. (1991), taken at the $3.6 \mathrm{~m}$ ESO telescope with the IDS in 1983, through a large circular aperture $8^{\prime \prime}$ in diameter. Unfortunately, the $[\mathrm{OIII}] \lambda 4363$ line was not detected in this galaxy, so direct determination of the electronic temperature, hence the Oxygen abundance, was not possible. We have therefore used the Pagel \& Edmunds (1983) fits, based on the $(\mathrm{OII}+\mathrm{OIII}) / \mathrm{H} \beta$ ratio, to estimate the $\mathrm{O} / \mathrm{H}$ abundance. Since the observational data fall in the double-valued region of these fits, we derive two values for the $\mathrm{O} / \mathrm{H}$ abundance: $12+\log (\mathrm{O} / \mathrm{H})=7.8$ (i.e. $0.08 Z_{\odot}$, assuming $\left.12+\log (\mathrm{O} / \mathrm{H})_{\odot}=8.9\right)$ and 8.36 (i.e. $\left.0.3 Z_{\odot}\right)$. We considered in principle both values for comparison with the predictions of synthesis models. Since the spectroscopic data provide only the $\mathrm{H} \beta$ flux, it is not possible to estimate properly the internal reddening from the ratios of Balmer emission lines. We have nevertheless verified that for $E(B-V)$ values below 0.50 , the resulting $\mathrm{O} / \mathrm{H}$ abundance is only weakly dependent on the reddening correction. We will see at the end that the extinction is indeed below $E(B-V)=0.4$.

Table 1 summarizes the observed photometric and spectroscopic data, as well as some derived parameters we will use in the analysis of Tol 0610-387. The data listed are corrected for Galactic extinction but not for internal reddening for which we do not have data.

\subsection{Analysis}

Spatially integrated (spectro-)photometry of BCDGs is difficult to interpret because it includes both the underlying galaxy and the starburst region. The contamination by older red giant stars, not related to the present burst of star formation, can affect significantly the IR colors. Similarly, the $\mathrm{H} \beta$ emission will be strongly contaminated 


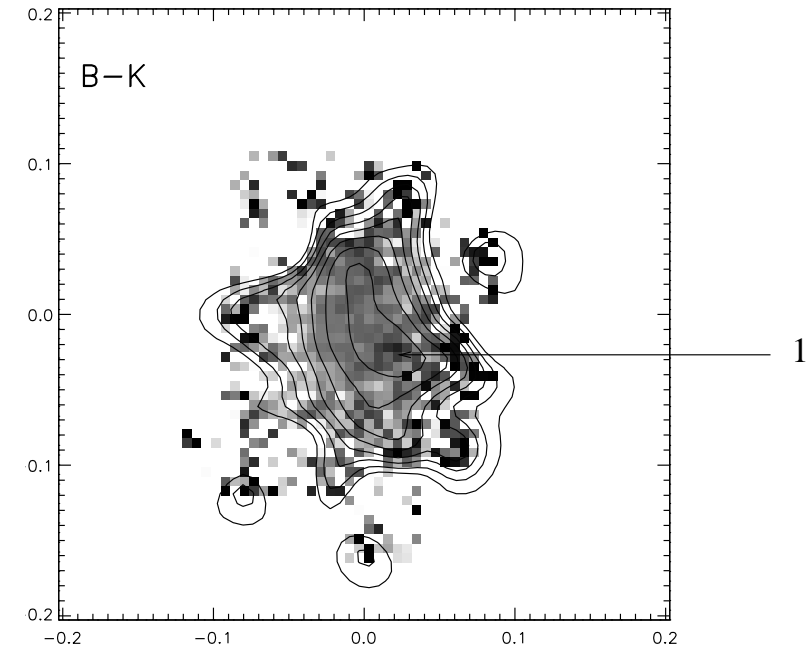

Fig. 4. Tol 0610-387 $B-K$ image. The outermost contour corresponds to $B-K=3$. The $B-K$ contour levels are spaced by 0.1 mag. In this case we only displayed the contours with a $S / N$ better than 3 . The region plotted roughly corresponds to region 1 only.

by the underlying $\mathrm{H} \beta$ absorbtion due to the same old stellar population, making it appear weaker. Therefore, while $W(\mathrm{H} \beta)$ will provide a strong constraint as an upper limit for the age of the burst, integrated spectro-photometry does not allow us to separate the contribution from the various "components" responsible for the colors: i.e. young red super giants, red giants, main sequence stars or nebular continuum. On the other hand, combining the spatial information provided by $2 \mathrm{D}$ color maps with $\mathrm{H} \alpha$ images allows us to disentangle the areas dominated by recent starbursts from the more evolved regions, making it possible to identify the regions over which the nebular emission is extended and to locate the different stellar populations.

We show in Fig. 1 the Tol 0610-387 $\mathrm{H} \alpha$ image, on which we have overplotted the $B$ band continuum contours, and in Fig. 2 the same $\mathrm{H} \alpha$ image with the $B-R$ contours overplotted. We have removed most of the artifacts due to image processing, so that only the significant data have been plotted. We note first that the maximum of all broad band continuum (including the $B$ - colors) does not coincide spatially with the maximum of $\mathrm{H} \alpha$ emission (see Fig. 2). Along the SW-NE direction we identify an incomplete arc of ionized regions surrounding the central area. The few HII knots appear embedded in a diffuse ionized cloud (grey shade in Figs. 1 and 2) offset with respect to the continuum maximum emission. This nuclear region corresponds geometrically to the center of the outer isophotes of the galaxy.

In Figs. 3 and 4 we have plotted the $J-K$ and $B-K$ maps corresponding to the central $25^{\prime \prime} \times 25^{\prime \prime}$ section. In both maps, a region of less than $5^{\prime \prime} \times 5^{\prime \prime}$ (about $45 \mathrm{pc}$ in projection for a galaxy distance of $18.8 \mathrm{Mpc}$ ) is clearly identifiable by its red $J-K$ and $B-K$ colors, spatially coincident with the maximum in $I$ and the minimum in
$B-R$ shown in Figs. 1 and 2. The $J-K$ map shows clearly that this central, red region is surrounded by a significantly bluer ring, which can be associated with the ionized blobs in Fig. 2. Unfortunately, the signal in $K$ ouside the plotted area is too weak to provide spatial information.

These color maps were derived directly from the broad band images. The CCD frames were co-aligned using the surrounding stars as reference for computing field rotation (between images in the visible and in the IR) and spatial offsets (Doublier et al. 1997).

From the analysis of the $\mathrm{H} \alpha, J-K$ and $B-K$ images, we have identified 3 extended regions showing very different integrated colors, which could be dominated by different stellar populations. We have marked in Fig. 3 the location of region 1 , the one potentially associated to the RSGs cluster as we will discuss later. Table 2 summarizes the average $B-R, B-K$ and $J-K$ colors of the three regions, as well as their $F(\mathrm{H} \alpha) / F$ ( $I$ band continuum) ratios. Since we did not have a narrow band image of the continuum near $\mathrm{H} \alpha$, the ratio $F(\mathrm{H} \alpha) / F(I)$ was derived from the combination of the $\mathrm{H} \alpha$ and the $I$ broad band images, giving a sort of $\mathrm{H} \alpha$ equivalent width. We stress that since the $\mathrm{H} \alpha$ image was not flux calibrated, the $F(\mathrm{H} \alpha) / F(I)$ ratios can only be used to analyze relative differences between various regions, but never as an absolute value. The integrated values have been obtained by averaging over the visually most representative areas on the images.

Table 2. Regions identified in Tol 0610-387.

\begin{tabular}{lllll}
\hline Region & $B-R^{*}$ & $B-K^{*}$ & $J-K^{*}$ & $F(\mathrm{H} \alpha) / F(I)^{* *}$ \\
\hline \#1 center region & 1.06 & 3.1 & 0.76 & 0.08 \\
\#2 semi-ring & 1.3 & 2.5 & {$[0.0,0.4]$} & 0.70 \\
\#3 outer regions & 3.1 & $\sim 3$. & $\sim-1.0$ & - \\
\hline
\end{tabular}

(*) No extinction correction has been applied to the color indices for better comparison with the color maps.

(**) Ratio between the $\mathrm{H} \alpha$ integrated flux (not calibrated) and the total flux in the $I$ passband, as explained in the text.

Region 1 is the central region clearly identified in Figs. 3 and 4 . It shows rather blue $B-R$ colors $((B-R) \sim 1)$, low $\mathrm{H} \alpha$ emission $(F(\mathrm{H} \alpha) / F(I)=0.08)$ and an excess in the $K$ band: $(B-K) \sim 3.1$, and $(J-K) \sim 0.8$.

Region 2, is constituted by the ring around the central region, including the $\mathrm{H} \alpha$ knots. $B-R$ increases $((B-R) \geq$ $1.3)$ and $\mathrm{H} \alpha$ peaks, with $(F(\mathrm{H} \alpha) / F(I)=0.70) . B-K$ and $J-K$ decrease $((B-K) \leq 2.5,(J-K) \sim 0.4)$.

Region 3 comprises the area with negligible $\mathrm{H} \alpha$ emission, as shown in Figs. 1 and 2, corresponding apparently to the underlying galaxy. It surrounds regions 1 and $2 . B-R$ becomes quite red, the $\mathrm{H} \alpha$ contribution becomes negligible and $B-K$ appears to increase again to $B-K \sim 3$, while 
$J-K$ decreases to -1.0 . We have to note that the galaxy is only marginally detected on the $K$ frames at radii larger than 10 arcsec, and that these colors have been obtained by integrating over larger areas.

\section{Discussion: Red supergiants in Tol 0610-387}

As explained above, the detection of ionized gas around a central stellar cluster showing very red $B-K$ and $J-K$ colors coupled with blue $B-R$ colors strongly suggests that we are looking at an evolved starburst which has developed an important population of red supergiant stars in its central region. The ionizing power in this region has already faded significantly, while some ionizing stars could still be present in the circumnuclear area, giving rise to the $\mathrm{H} \alpha$ knots and diffuse emission detected there and to its bluer continuum colors. Assuming this scenario we have compared the measured infrared colors with the predictions of evolutionary synthesis models from Cerviño \& Mas-Hesse (1994). Throughout the analysis, we have assumed a Salpeter Initial Mass Function (i.e. with a slope of -2.35), with upper and lower mass limits at 120 and $2 M_{\odot}$, respectively. The results do not change significantly with different values of the IMF slope. As explained in Mas-Hesse \& Kunth (1991), the inclusion of lower mass stars does not affect significantly the predicted colors. We have compared our observational data with the models computed for $Z=0.4 Z_{\odot}$ and $Z=0.05 Z_{\odot}$. The first one is close to one of the measured abundance values $\left(Z \approx 0.3 Z_{\odot}\right)$, while the other one is representative of low metallicities scenarios. The $Z=0.4 Z_{\odot}$ model has been selected over the one at $Z=0.2 Z_{\odot}$ since the formation of red supergiant stars is critically dependent on metallicity at very low abundances, and an underestimate of the metallicity can lead to a severe underestimate of the RSG population.

Region 1 (post-starburst area): We have plotted in Fig. 5 the evolution of the predicted $B-K, J-K$ and $B-R$ colors as a function of time for the two metallicities considered. The models by Cerviño \& Mas-Hesse (1994) predict that, at a metallicity of around $0.4 Z_{\odot}$, red supergiant stars begin to dominate the infrared emission 6 Myrs after the onset of the starburst, fading at around 15 Myrs. After 15 Myr RSGs are still being produced, but their contribution to the total luminosity of the cluster and its colors becomes progressively smaller. The presence of massive RSGs during this period produces a broad peak in $B-K$, with values up to $B-K \approx 2.2$ at around 10-15 Myrs. The effect is weaker in the $B-R$ and $J-K$ colors. At very low metallicities, on the other hand, the population of RSGs is so small that it does not affect significantly the integrated colors, which are much bluer at any age. Evolutionary synthesis models by Schaerer \& Vacca (1998), which assume enhanced mass loss rates with respect to the ones by Cerviño \& Mas-Hesse (1994), show a similar evolution with time of the $B-K$ color, but with a higher peak value $B-K \approx 2.8$.

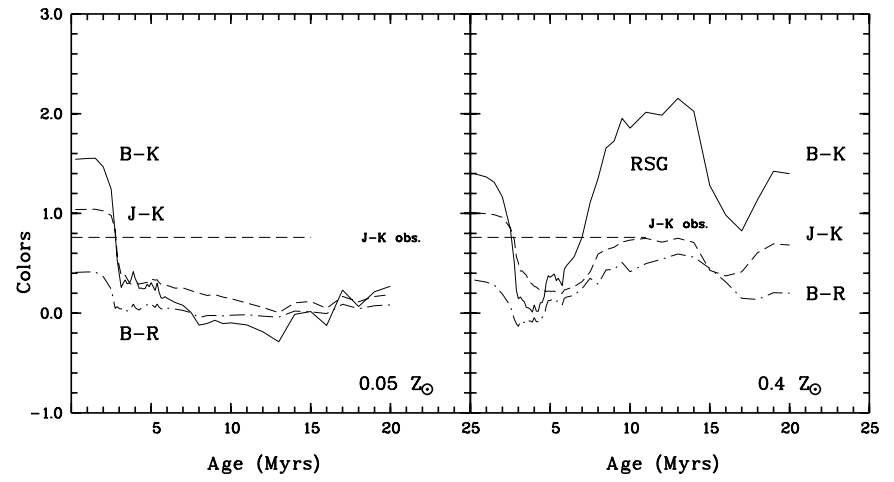

Fig. 5. Comparison with evolutionary synthesis models. The left panel represents the predicted evolution of the $B-R, J-K$ and $B-K$ colors as functions of the age of the starburst for a metallicity of $0.05 Z_{\odot}$. The right panel represents the same predicted colors for a metallicity of $0.4 Z_{\odot}$.

Comparing the colors listed in Table 2 with the predictions of the models we find that:

- The $J-K$ color, which is only weakly sensitive to internal or external extinction, would be consistent with the predictions of low metallicity models only within the first $3 \mathrm{Myr}$. At this age the $K$ band is dominated by nebular continuum emission (Cerviño \& Mas-Hesse 1994). In Fig. 1, the $\mathrm{H} \alpha$ emission is rather weak in the central region of the galaxy, while the continuum emission peaks. We conclude that the observations are not consistent with a very low metallicity scenario. We will therefore neglect this lower abundance value in the following discussion;

The fact that the $F(\mathrm{H} \alpha) / F$ ( $I$ band continuum) ratio is much weaker in region 1 than in the ring surrounding it (region 2), entails the impossibility that the weaker $\mathrm{H} \alpha$ emission in region 1 could be due to enhanced extinction in this area, since both $\mathrm{H} \alpha$ and $I$ continuum would be affected in a similar way. Similarly, we conclude that the observed morphology is not a result of dust distribution;

- A continuous star formation process would have produced new generations of massive, ionizing stars which apparently are not present in the central cluster (the $\mathrm{H} \alpha$ emission in the central region is rather weak). We have therefore neglected this kind of models;

- The observed $J-K$ value is consistent with the predictions for a nearly instantaneous burst at intermediate metallicity $\left(Z \approx 0.4 Z_{\odot}\right)$, with an age in the range 8-14 Myr. Above $8 \mathrm{Myr}$, the $K$ emission from a young massive cluster is dominated by red supergiant stars. From the luminosity of the central region in the $K$ band, we could roughly estimate the number of RSG: the absolute $K$ mag of the central region is -16.4 , while a single RSG has an absolute $K$ mag of about -10.5 , hence the number of RSGs present in the central cluster would be around 250 stars. 
Both $B-K$ and $B-R$ colors are redder than predicted by the models at any age. This is probably due to the effect of internal reddening, which would affect mostly the $B$ flux, but the $K$ flux only weakly. If we correct the predicted colors from total reddening in order to fit the observed ones, we derive consistent values of total extinction: $E(B-$ $V)=0.32$ and 0.38 for $B-K$ and $B-R(J-K$ is almost insensitive to reddening). We have used for this the (Savage \& Mathis 1979) Galactic extinction law, and the model predictions at $12 \mathrm{Myr}$. A consistent but worse fit would also be obtained around $20 \mathrm{Myr}$, with $E(B-V)$ values of 0.45 and 0.58 for $B-K$ and $B-R$.

The weakness, but not absence, of $\mathrm{H} \alpha$ emission in the central region is also consistent with an evolved cluster around 10-15 Myr old. Synthesis models predict $W(\mathrm{H} \beta)$ values around $2 \AA$ at $12 \mathrm{Myr}$, and around $6 \AA$ at $9 \mathrm{Myr}$, but the ionizing power decreases very rapidly with time, yielding only $0.2 \AA$ at $20 \mathrm{Myr}$. Therefore, models at ages around 20 Myr have also been neglected. On the other hand, comparison of the observed $W(\mathrm{H} \beta)$ values with the predictions of models does not rule out that the central starburst could be younger, if the contamination of the continuum at $4861 \AA$ by underlying, older stars is significant. Unfortunately, not having better spectroscopical data, we can noy use the $W(\mathrm{H} \beta)$ value for a more accurate determination of age. We want just to stress that values around $5 \pm 5 \AA$ are consistent with evolved clusters at ages where the most massive stars have already evolved into RSGs.

Region 2: The relatively low $\mathrm{H} \beta$ equivalent width measured by Terlevich et al. (1991) corresponds to a circular aperture $8^{\prime \prime}$ in diameter, i.e., comprising only region 1 as marked in Fig. 3. Higher values are expected on the knots of region 2, as indicated by the $F(\mathrm{H} \alpha) / F(I)$ ratios, suggesting a younger episode of star formation there, possibly triggered by the central starburst. The $J-K$ color measured in region 2 would be indeed consistent with a starburst around 4-7 Myr old. The ring structure around the central cluster suggests strongly that the star formation has migrated outwards. Possibly, it was tiggered by the explosion of the central starburt $\mathrm{SNe}$, as observed in 30 Doradus (de Boer 1998).

Region 3: The very red colors (both in the optical and in the IR) and the lack of $\mathrm{H} \alpha$ emission are characteristic of an evolved population of stars. We compared the observed colors directly with the calibrations made by Koornneef (1983), and Bessel \& Brett (1998) for standard stars of various spectral types. We find that the colors are consistent with a population dominated by red giant stars of late $K$ and early $\mathrm{M}$ spectral types. The colors are definitely not consistent with main-sequence stars or supergiants. These stars (possibly population II) are at least 5-10 billion years old and cannot be associated with a young population. Their presence rules out any suggestion of "youth" for this galaxy.

The consistency of the measured colors with the predictions of synthesis models for the scenario we have assumed, strongly suggests that we might be looking at a stellar cluster dominated by red supergiant stars. We conclude therefore that the large values of $B-K$ and $J-K$ in the central cluster could be associated to the population of RSGs expected for a starburst between 10 to 15 Myr old. Confirmation of the presence of RSGs should be obtained by spectroscopic analysis in the IR, or direct imaging using space telescopes or the VLT under excellent seeing conditions. Our results make Tol 0610-387 a preferred candidate for follow-up spectroscopic search of RSG characteristic features.

\section{Conclusions}

$\mathrm{H} \alpha$ imaging combined with $B-K$ and $J-K$ mapping has allowed us to identify stellar clusters with an excess of $K$ emission close to regions with recent star-formation activity in the BCDG Tol 0610-387. Comparison with the predictions of evolutionary population synthesis models suggests that the observed colors originate from a population of around 250 red supergiant stars, evolved in a nuclear starburst having taken place around 10-15 Myr ago, and whose ionizing power has faded almost completely in the present time. More recent star formation knots (4-7 Myr old) have been identified in circumnuclear areas, possibly triggered by the central starburst.

Our results provide a strong hint on the presence of RSGs in this kind of BCDGs hosting relatively evolved starbursts episodes associated possibly with migrating star formation. These galaxies potentially hosting a relatively large number of red supergiants stars become key targets for follow-up spectroscopic identification by analysis of the Ca triplet and the CO index. The spectroscopic confirmation of the presence of RSGs in these galaxies would allow us to analyze in detail the relations between the dynamics of the starburst and the host galaxy, by comparing the velocity dispersions of the gas with that of the stars.

Acknowledgements. This work has been partially supported by Spanish CICYT under grant ESP95-0389-C02-02.

\section{References}

Ambrocio-Cruz, P., Laval, A., Marcelin, M., \& Amram, P. 1997, A\&A, 319, 973

Bessel, M., \& Brett, J. 1998, PASP, 100, 1134

Burstein, D., \& Heiles, C. 1982, AJ, 87, 1165

Campbell, A. W., \& Terlevich, R. 1984, MNRAS, 211, 15

Cerviño, M., \& Mas-Hesse, J. M. 1994, A\&A, 284, 749

Chu, Y.-H., \& Kennicutt, Robert, C. J. 1994, ApJ, 425, 720

de Boer, K. S. 1998, in The Magellanic Clouds and Other Dwarf Galaxies, Proceedings of the Bonn/Bochum-Graduiertenkolleg Workshop, held at the Physikzentrum Bad Honnef, Germany, January 19-22, 1998, ed. T. Richtler, \& J. M. Braun (Shaker Verlag, Aachen, ISBN 3-8265-4457-9), 125

Doublier, V., Caulet, A., \& Comte, G. 1999, A\&AS, 138, 213

Doublier, V., Caulet, A., \& Comte, G. 2001, A\&A, 367, 33 
Doublier, V., Comte, G., Petrosian, A., Surace, C., \& Turatto, M. 1997, A\&AS, 124, 405

Hunter, D. A., \& Thronson, Harley, A. J. 1995, ApJ, 452, 238 Izotov, Y. I., Dyak, A. B., Chaffee, F. H., \& et al. 1996, ApJ, 458,524

Koornneef, J. 1983, A\&A, 128, 84

Landolt, A. U. 1992, AJ, 104, 340

Legrand, F., Kunth, D., Mas-Hesse, J. M., \& Lequeux, J. 1997, A\&A, 326, 929

Lequeux, J., Kunth, D., Mas-Hesse, J. M., \& Sargent, W. L. W. 1995, A\&A, 301, 18

Mas-Hesse, M., \& Kunth, D. 1999, A\&A, 349, 765

Mas-Hesse, J. M., \& Kunth, D. 1991, A\&AS, 88, 399

Massey, P. 1998, ApJ, 501, 153

Massey, P., Lang, C. C., Degioia-Eastwood, K., \& Garmany, C. D. 1995, ApJ, 438, 188
Melnick, J., Moles, M., Terlevich, R., \& Garcia-Pelayo, J.-M. 1987, MNRAS, 226, 849

Meurer, G. R., Heckman, T. M., \& Leitherer, C., et al. 1995, AJ, 110, 2665

Pagel, B. E. J., \& Edmunds, M. G. 1983, Nature, 304, 488

Roy, J.-R., \& Belley, J. 1993, ApJ, 406, 60

Savage, B. D., \& Mathis, J. S. 1979, ARA\&A, 17, 73

Schaerer, D., \& Vacca, W. D. 1998, ApJ, 497, 618

Terlevich, R., Melnick, J., Masegosa, J., Moles, M., \& Copetti, M. V. F. 1991, A\&AS, 91, 285

Thuan, T. X., Izotov, Y. I., \& Lipovetsky, V. A. 1996, ApJ, 463,120

Zinnecker, H. 1987, in Starbursts and galaxy evolution; Proceedings of the 22nd Moriond Astrophysics Meeting, Les Arcs, France (Éditions Frontières), 165 